\title{
The fate of the pre-main sequence-rich clusters Collinder 197 and vdB 92: dissolution?
}

\author{
C. Bonatto ${ }^{1}$ and E. Bica ${ }^{1}$ \\ Universidade Federal do Rio Grande do Sul, Departamento de Astronomia, CP 15051, RS, Porto Alegre 91501-970, Brazil
}

e-mail: [charles;bica]@if.ufrgs.br

Received 27 January 2010 / Accepted 16 March 2010

ABSTRACT

\begin{abstract}
Context. Since most of the star clusters do not survive the embedded phase, their early dissolution appears to be a major source of field stars. However, catching a cluster in the act of dissolving is somewhat elusive.

Aims. We investigate the nature and possible evolution of the young Galactic star clusters Collinder 197 (Cr 197) and vdB 92.

Methods. Photometric and structural properties were derived with near-infrared photometry and field-star decontamination. Kinematical properties were inferred from proper motions of the main sequence (MS) and pre-MS (PMS) member stars.

Results. The colour-magnitude diagrams (CMDs) are basically characterised by a poorly-populated MS and a dominant fraction $(\gtrsim 75 \%)$ of PMS stars, and the combined MS and PMS CMD morphology in both clusters consistently constrains the age to within $5 \pm 4 \mathrm{Myr}$, with a $\sim 10 \mathrm{Myr}$ spread in the star formation process. The MS+PMS stellar masses are $\approx 660_{-59}^{+102} M_{\odot}(\mathrm{Cr} 197)$ and $\approx 750_{-51}^{+101} M_{\odot}(\mathrm{vdB} 92)$. Both $\mathrm{Cr} 197$ and vdB 92 appear to be abnormally large when compared to clusters within the same age range. They have irregular stellar radial density distributions (RDPs) with a marked excess in the innermost region, a feature that, at less than $10 \mathrm{Myr}$, is more likely related to the star formation and/or molecular cloud fragmentation than to age-dependent dynamical effects. The velocity dispersion of both clusters, derived from proper motions, is in the range $\sim 15-22 \mathrm{~km} \mathrm{~s}^{-1}$.

Conclusions. Both clusters appear to be in a super-virial state, with velocity dispersions higher than those expected of nearly-virialised clusters of similar mass and size. A possible interpretation is that $\mathrm{Cr} 197$ and vdB 92 deviate critically from dynamical equilibrium, and may dissolve into the field. We also conclude that early cluster dissolution leaves detectable imprints on RDPs of clusters as massive as several $10^{2} M_{\odot}$. $\mathrm{Cr} 197$ and vdB 92 may be the link between embedded clusters and young stellar associations.
\end{abstract}

Key words. open clusters and associations: general - open clusters and associations: individual: Collinder 197 open clusters and associations: individual: vdB 92

\section{Introduction}

It has only now become well-established that a significant fraction of the embedded star clusters dissolve into the field on a time scale of a few $10^{7}$ Myr. Basically, dissolution occurs mainly because the gravitational potential can be rapidly reduced by internal processes, such as the impulsive gas removal by supernovae and massive star winds associated with this early period. As a consequence, an important fraction of the stars, especially with low mass, end up moving faster than the scaled-down escape velocity and may be lost to the field (e.g. Goodwin \& Bastian 2006). This process can dissolve the very young star clusters on time scales of 10-40 Myr.

From the stellar content perspective, the early cluster dissolution depends essentially on the effective star-formation efficiency, the total mass converted into stars, and the mass of the more massive stars (e.g. Tutukov 1978; Goodwin \& Bastian 2006). However, there is also evidence that the determining factor for cluster survival during gas expulsion is the virial state of the stars just before the onset of this phase, so that clusters formed with a dynamically cold stellar component are more likely to survive (Goodwin 2009).

In any case, the early dissolution of embedded clusters may lead to the formation of OB stellar groups (e.g. Gouliermis et al. 2000), the subsequent dispersion of which may be an important source of field stars (e.g. Massey et al. 1995). Indeed, Lada \& Lada (2003) suggest that only about $5 \%$ of the
Galactic embedded clusters dynamically evolve into gravitationally bound open clusters (OCs). With such a high dissolution rate, the embedded clusters could be the major contributors of field stars in galaxies for generations. However, recent studies suggest that the early dissolution rate in the Magellanic Clouds is significantly lower ( $\$ 30 \%$ - de Grijs \& Goodwin 2008, 2009) than in the Milky Way (Lada \& Lada 2003) or in other galaxies such as the Antennae (e.g. 2007) or M51 (e.g. Bastian et al. 2005).

Observationally, low-mass star clusters younger than $\sim 10 \mathrm{Myr}$, in general, have colour-magnitude diagrams (CMDs) with an under-populated and developing main sequence (MS) and a more conspicuous population of pre-MS (PMS) stars. Typical examples are NGC 6611, NGC 4755, NGC 2239, NGC 2244, Bochum 1, Pismis 5, NGC 1931, vdB 80, and BDBS 96 (Bonatto \& Bica 2009c, and references therein). In terms of CMD morphology, bound and unbound young clusters are expected to present similar evolutionary sequences. On the other hand, the important early changes in the potential that affect the large-scale internal structure of clusters should be reflected in the stellar radial density profile (RDP). Bochum 1 (Bica et al. 2008a), and NGC 2244 (Bonatto \& Bica 2009b), for instance, appear to be representatives of this scenario (i.e. structures evolving towards dissolution in a few $10^{7} \mathrm{yr}$ ), in which an irregular RDP cannot be represented by a cluster-like profile (i.e. an approximately isothermal sphere). In this context, irregular 
RDPs in young clusters - when coupled to an abnormally highvelocity dispersion - may reflect significant profile erosion or dispersion of stars, and point to significant deviations from dynamical equilibrium.

The rather complex interplay among environment conditions, effective star-formation efficiency (as defined in Goodwin \& Bastian 2006), and total mass converted into stars, is probably what explains the difference between (dissolving) objects like Bochum 1 and NGC 2244, and bound young OCs (in which the MS+PMS stars distribute according to a cluster RDP as in NGC 6611 and NGC 4755). Consistent with the above mass-dependent scenario, the MS+PMS mass of Bochum 1 and NGC 2244 is a factor 2-3 lower than in NGC 6611 and NGC 4755.

In this paper we investigate the nature of the poorly-studied, young (age $~ 5$ Myr; Sect. 4), large (with radii within 8-12 pc; Sect. 5), and PMS-rich (with stellar masses within 660-750 $M_{\odot}$; Sect. 6) clusters Cr 197 and vdB 92. Our main goal is to determine whether such young stellar systems can be characterised as typical OCs or if they are heading towards dissolution. In addition, we derive their fundamental and structural parameters, most of these for the first time.

This paper is organised as follows. In Sect. 2 we recall the literature data on both objects. In Sect. 3 we discuss the 2MASS photometry and build the field-star decontaminated CMDs. In Sect. 4 we derive fundamental cluster parameters. In Sect. 5 we derive structural parameters. In Sect. 6 we estimate cluster mass. In Sect. 7 we build the intrinsic proper motion distribution. In Sect. 8 we compare structural parameters and dynamical state with those of a sample of template OCs. Concluding remarks are given in Sect. 9.

\section{Previous data on $\mathrm{Cr} 197$ and vdB 92}

Collinder 197 (Cr 197) was discovered by Collinder (1931) in Vela. It was also listed in the OC catalogue of Alter et al. (1970) as OCl-742, and the ESO/Uppsala Blue Plate Southern (Lauberts 1982) as ESO $313 \mathrm{SC} 13$.

Vogt \& Moffat (1973) centred the cluster on the B4 II star HIP 42908 at $\alpha(2000)=08^{\mathrm{h}} 44^{\mathrm{m}} 40.3^{\mathrm{s}}, \delta(2000)=-41^{\circ} 16^{\prime} 38^{\prime \prime}$. This star, which is reported as a double system in SIMBAD, is the brightest in the area with $V=7.32$. HIP 42908 may be the cluster centre in the infrared, since strong absorption is suggested by the B plate image seen to the southwest of HIP 42908. Vogt \& Moffat (1973) found the reddening $E(B-V)=0.58$ and the distance from the Sun $d_{\odot}=1.05 \mathrm{kpc}$.

Besides dust absorption and emission in the area, Cr 197 is also embedded in the HII region Gum 15 (Gum 1955), also known as RCW 32 (Rodgers et al. 1960). Assuming that HIP 42908 is a cluster member, it might be responsible for the emission. Indeed, Pettersson \& Reipurth (1994) have identified lowluminosity emission-line stars in $\mathrm{Cr} 197$ and in the R-association Vela R2, which may be part of their low-mass population. They derive $d_{\odot}=1.1 \mathrm{kpc}$ and estimate a few $10^{6} \mathrm{yr}$ of age. Battinelli \& Capuzzo-Dolcetta (1991) and Battinelli et al. (1994) estimated 6.3 Myr of age, $d_{\odot}=1 \mathrm{kpc}$, a total mass of $\approx 100 M_{\odot}$, and the integrated absolute magnitude $M_{V}=-5.16$.

Found by van den Bergh (1966), the embedded cluster $\mathrm{vdB} 92$ is related to the reflection nebula vdB-RN 92, which is located in Canis Major. The cluster is also listed as FSR 1188 by Froebrich et al. (2007) who, based on the $H$ photometry, derived the core and tidal radii $R_{\mathrm{c}, H}=1.1^{\prime}$ and $R_{\mathrm{t}, H}=24^{\prime}$, and the total number of members $N=297$ stars. Magakian (2003) also reports the reflection nebula and related cluster. It is immersed
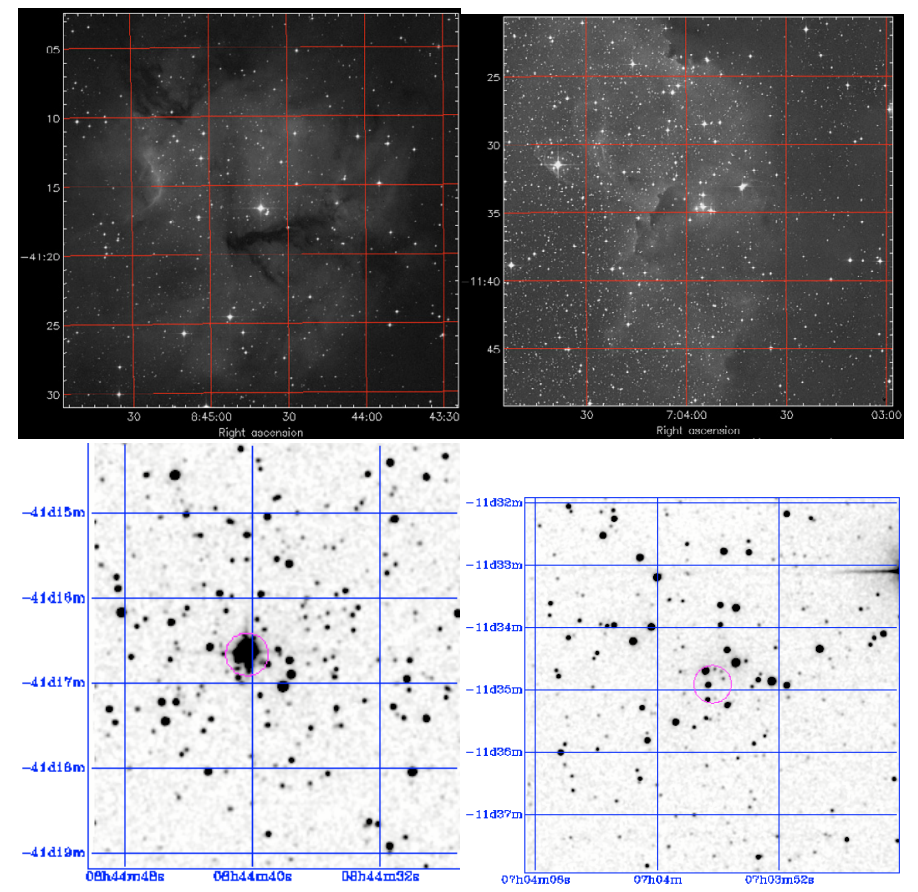

Fig. 1. Top: $20^{\prime} \times 20^{\prime}$ DSS-II R images centred on Cr 197 (left) and vdB 92 (right). Gas emission, dust reflection, and/or absorption are present in the fields in varying proportions. Bottom: 2 MASS $K_{\mathrm{s}}$ images covering $5^{\prime} \times 5^{\prime}(\mathrm{Cr} 197)$ and $6^{\prime} \times 6^{\prime}(\mathrm{vdB} 92)$. Orientation: North at the top and east to the left.

in the large angular size association CMa OB1 (Clariá 1974a,b) and in the reflection nebula association CMa R1 (van den Bergh 1966). The equally extended emission nebula IC 2177, or Gum 2 (Gum 1955), seems to permeate the complex. The relatively bright star at $\approx 3.4^{\prime}$ to the northeast of the cluster vdB 92 is the Herbig Be star Z CMa, which has a bipolar outflow (Poetzel et al. 1989). Undoubtedly, vdB 92 is part of a star-forming complex that deserves further analyses. With an approach that differs in various aspects from the present one, Soares \& Bica (2003) studied the brighter sequences of vdB 92, deriving an age of 5-7 Myr, a mean visible absorption $A_{\mathrm{v}}=4.4$, and $d_{\odot} \approx 1.5 \mathrm{kpc}$.

A few bright stars mixed with nebular gas and/or dust emission are seen in the $20^{\prime} \times 20^{\prime} \mathrm{R}$ images (Fig. 1, taken from LEDAS $^{1}$ ). Close-ups of the clusters are shown in the smaller field 2MASS $K_{\mathrm{s}}$ images. Table 1 provides parameters found in the literature and derived here. The central coordinates were computed again to match the absolute maxima present in the stellar surface densities (Sect. 3.2). The re-computed central coordinates of Cr 197 are almost coincident with HIP 42908.

\section{Construction of decontaminated CMDs}

Since both objects still retain part of the primordial gas and dust (Fig. 1), the near-IR provides the adequate depth to study them, especially the faint stellar sequences. For this purpose, we work with the $2 \mathrm{MASS}^{2} J, H$, and $K_{\mathrm{s}}$ photometry, which provides the spatial and photometric uniformity required for wide extractions that, in turn, result in high star-count statistics.

\footnotetext{
${ }^{1}$ Leicester Database and Archive Service (LEDAS) DSS/DSS-II service on ALBION; http://ledas-www.star.le.ac.uk/DSSimage 2 The Two Micron All Sky Survey, All Sky data release (Skrutskie et al. 1997) - http://www.ipac.caltech.edu/2mass/releases/ allsky/
} 
Table 1. Fundamental parameters.

\begin{tabular}{|c|c|c|c|c|c|c|c|c|c|c|c|c|c|c|}
\hline \multirow[b]{2}{*}{ Cluster } & \multicolumn{6}{|c|}{ WEBDA } & \multicolumn{8}{|c|}{ This work } \\
\hline & $\begin{array}{c}\alpha(2000) \\
(\mathrm{hms})\end{array}$ & $\begin{array}{c}\delta(2000) \\
\left({ }^{\circ}{ }^{\prime \prime \prime}\right)\end{array}$ & $\begin{array}{l}\text { Age } \\
(\mathrm{Myr})\end{array}$ & $\begin{array}{c}E(B-V) \\
(\mathrm{mag})\end{array}$ & $\begin{array}{c}d_{\odot} \\
(\mathrm{kpc})\end{array}$ & $\begin{array}{l}D \\
\left({ }^{\prime}\right)\end{array}$ & $\begin{array}{c}\alpha(2000) \\
(\mathrm{hms})\end{array}$ & $\begin{array}{c}\delta(2000) \\
\left({ }^{\prime \prime \prime \prime}\right)\end{array}$ & $\begin{array}{l}\ell \\
\left({ }^{\circ}\right)\end{array}$ & $\begin{array}{l}b \\
\left({ }^{\circ}\right)\end{array}$ & $\begin{array}{l}\text { Age } \\
(\mathrm{Myr})\end{array}$ & $\begin{array}{c}E(B-V) \\
(\mathrm{mag})\end{array}$ & $\begin{array}{c}d_{\odot} \\
(\mathrm{kpc})\end{array}$ & $\begin{array}{l}\Delta R_{\mathrm{SC}} \\
(\mathrm{kpc})\end{array}$ \\
\hline (1) & (2) & (3) & (4) & (5) & (6) & (7) & (8) & (9) & (10) & (11) & (12) & (13) & (14) & (15) \\
\hline Cr 197 & $08: 44: 51$ & $-41: 14: 00$ & 13 & 0.55 & 0.84 & 17.0 & $08: 44: 40.3$ & $-41: 16: 48.4$ & 261.51 & +0.94 & $5 \pm 4$ & $0.34 \pm 0.16$ & $1.05 \pm 0.20$ & $0.23 \pm 0.04$ \\
\hline vdB 92 & 07:03:54 & $-11: 29: 23$ & 40 & 0.25 & 1.50 & 24.0 & 07:03:56.4 & $-11: 34: 54.7$ & 224.66 & -2.52 & $5 \pm 4$ & $0.22 \pm 0.13$ & $1.38 \pm 0.26$ & $1.04 \pm 0.19$ \\
\hline
\end{tabular}

Notes. Column 7: optical diameter; Col. 14: distance from the Sun; Col. 15: distance from the Solar circle. WEBDA data for Cr 197 is based on photometry from Vogt \& Moffat (1973).

Table 2. Derived structural parameters.

\begin{tabular}{ccccccccccc}
\hline \hline Cluster & $\begin{array}{c}\sigma_{\mathrm{bg}} \\
\left(*^{\prime-2}\right)\end{array}$ & $\begin{array}{c}\sigma_{0} \\
\left(*^{\prime-2}\right)\end{array}$ & $\begin{array}{c}R_{\mathrm{c}} \\
\left({ }^{\prime}\right)\end{array}$ & $\begin{array}{c}R_{\mathrm{RDP}} \\
\left({ }^{\prime}\right)\end{array}$ & $\delta_{\mathrm{c}}$ & $\begin{array}{c}1^{\prime} \\
(\mathrm{pc})\end{array}$ & $\begin{array}{c}\sigma_{\mathrm{bg}} \\
\left(* \mathrm{pc}^{-2}\right)\end{array}$ & $\begin{array}{c}\sigma_{0} \\
\left(* \mathrm{pc}^{-2}\right)\end{array}$ & $\begin{array}{c}R_{\mathrm{c}} \\
(\mathrm{pc})\end{array}$ & $\begin{array}{c}R_{\mathrm{RDP}} \\
(\mathrm{pc})\end{array}$ \\
$(1)$ & $(2)$ & $(3)$ & $(4)$ & $(5)$ & $(6)$ & $(7)$ & $(8)$ & $(9)$ & $(10)$ & $(11)$ \\
\hline $\mathrm{Cr} 197^{\dagger}$ & $2.17 \pm 0.02$ & $5.0 \pm 1.0$ & $4.8 \pm 0.8$ & $40.0 \pm 4.0$ & $3.3 \pm 0.5$ & 0.305 & $23.3 \pm 0.1$ & $97.9 \pm 20.9$ & $1.5 \pm 0.3$ & $12.2 \pm 1.2$ \\
$\mathrm{Cr} 197^{\ddagger}$ & $2.15 \pm 0.04$ & $4.7 \pm 1.0$ & $5.2 \pm 0.9$ & $40.0 \pm 4.0$ & $3.2 \pm 0.5$ & 0.305 & $23.1 \pm 0.2$ & $92.8 \pm 17.9$ & $1.6 \pm 0.3$ & $12.2 \pm 1.2$ \\
$\mathrm{vdB} 9^{\dagger}$ & $2.01 \pm 0.06$ & $2.2 \pm 0.7$ & $4.8 \pm 1.3$ & $20.0 \pm 2.0$ & $2.1 \pm 0.3$ & 0.402 & $12.5 \pm 0.4$ & $13.6 \pm 4.4$ & $1.9 \pm 0.5$ & $8.0 \pm 0.8$ \\
$\mathrm{vdB} 92^{\ddagger}$ & $1.97 \pm 0.06$ & $2.7 \pm 0.6$ & $5.0 \pm 0.9$ & $20.0 \pm 2.0$ & $2.4 \pm 0.3$ & 0.402 & $12.3 \pm 0.4$ & $17.0 \pm 3.6$ & $2.0 \pm 0.4$ & $8.0 \pm 0.8$ \\
\hline
\end{tabular}

Notes. Column 6: cluster/background density contrast parameter $\left(\delta_{\mathrm{c}}=1+\sigma_{0} / \sigma_{\mathrm{bg}}\right)$ computed with the King-like fit parameters; Col. 7: arcmin to parsec scale. $\dagger$ background level kept fixed; $¥$ Background level allowed to vary.

Our group has been developing analytical tools for 2MASS photometry that allow us to statistically disentangle cluster evolutionary sequences from field stars in CMDs. Decontaminated CMDs have been used to investigate the nature of star cluster candidates and derive their astrophysical parameters. In summary, we applied (i) field-star decontamination to uncover the intrinsic CMD morphology, essential for a proper derivation of reddening, age, and distance from the Sun, and (ii) colour-magnitude filters, which are required for intrinsic stellar RDPs, as well as luminosity and mass functions (MFs). In particular, the use of field-star decontamination in the construction of CMDs has proved to constrain age and distance more than the raw (observed) photometry, especially for low-latitude and/or bulge-projected OCs (e.g. Bica et al. 2008b, and references therein).

Photometry for both clusters was extracted from Vizie ${ }^{3}$ in a wide circular field of radius $R_{\text {ext }}=80^{\prime}$, which is adequate for determining the background level (Sect. 5) and statistically characterising the colour and magnitude distribution of the field stars (Sect. 3.1). As a photometric quality constraint, only stars with $J, H$, and $K_{\mathrm{s}}$ errors lower than $0.1 \mathrm{mag}$ were used. Reddening corrections were based on the absorption relations $A_{J} / A_{V}=0.276, A_{H} / A_{V}=0.176, A_{K_{\mathrm{S}}} / A_{V}=0.118$, and $A_{J}=2.76 \times E(J-H)$ given by Dutra et al. (2002), with $R_{V}=3.1$, considering the extinction curve of Cardelli et al. (1989). The reddening values were derived from the 2MASS CMDs (Sect. 4).

\subsection{Field decontamination}

Field-star decontamination is usually required for proper identification and characterisation of star clusters, especially those near the Galactic equator and/or with large fractions of faint stars. Cr 197 and vdB 92 are located in the 3rd Galactic quadrant (Table 1), which makes field-star contamination a minor

\footnotetext{
3 http://vizier.u-strasbg.fr/viz-bin/VizieR? - source=II/246
}

issue (e.g. Bica et al. 2008b). However, their CMDs are dominated by PMS stars (Sect. 4), so, decontamination is important for avoiding confusion with the red dwarfs of the Galactic field. A summary of different decontamination approaches is provided in Bonatto \& Bica (2009b). In this paper we apply the decontamination algorithm developed in Bonatto \& Bica (2007), together with an improvement that is described below.

Photometric uncertainties are explicitly taken into account, in the sense that stars have a non-negligible probability (assumed to be Gaussian) of having a magnitude and colour significantly different from the average values. The algorithm (i) divides the full range of magnitude and colours of a CMD into a $3 \mathrm{D}$ grid of cells with axes along the $J$ magnitude and the $(J-H)$ and $\left(J-K_{\mathrm{s}}\right)$ colours, (ii) computes the total (i.e. member+field) number density of stars within a given cell $\left(\eta_{\text {tot }}\right)$, (iii) estimates the number density of field stars $\left(\eta_{\mathrm{fs}}\right)$ based on the number of comparison field stars with similar magnitude and colours as those in the cell, (iv) computes the expected number density of member stars, $\eta_{\mathrm{mem}}=\eta_{\mathrm{tot}}-\eta_{\mathrm{fs}},(v)$ converts the number density $\eta_{\mathrm{fs}}$ into the estimated number of field stars and subtracts it from each cell, and ( $v i)$ after subtraction of the field stars, the remaining $N_{\text {clean }}^{\text {cell }}$ stars in each cell are identified for further use (see below). The wide annulus between $50^{\prime}<R<80^{\prime}$ (outside the cluster radius - Table 2) is used as the comparison field for $\mathrm{Cr} 197$; for vdB 92, it is located between $30^{\prime}<R<80^{\prime}$. The initial cell dimensions are $\Delta J=1.0$ and $\Delta(J-H)=\Delta\left(J-K_{\mathrm{s}}\right)=0.2$, but cell sizes half and twice the initial values are also used. As a new feature, we also consider shifts in the grid positioning by $\pm 1 / 3$ of the respective cell size in the 2 colours and magnitude axes. When all the different grid/cell size setups are applied, the decontamination take 729 independent combinationss into account.

Each setup produces a total number of member stars, $N_{\text {mem }}=$ $\sum_{\text {cell }} N_{\text {clean }}^{\text {cell }}$, from which we compute the expected total number of member stars $\left\langle N_{\text {mem }}\right\rangle$ by averaging out $N_{\text {mem }}$ over all combinations. Stars (identified in step ( $v i)$ above) are ranked according to the number of times they survive all runs (survival frequency), 


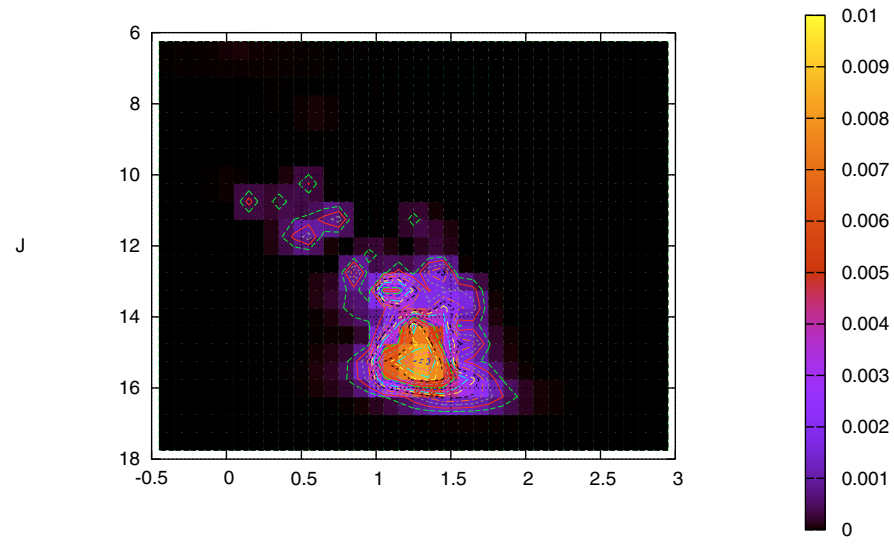

$(\mathrm{J}-\mathrm{Ks})$

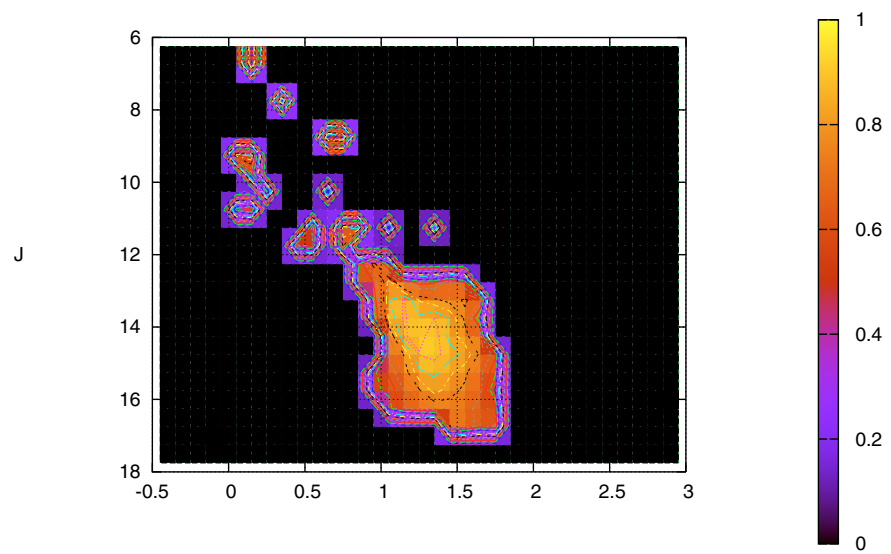

$(\mathrm{J}-\mathrm{Ks})$

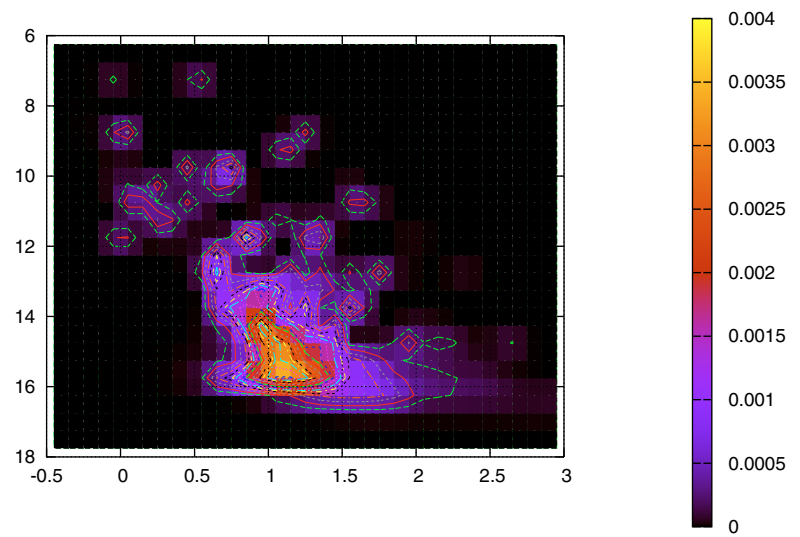

$(\mathrm{J}-\mathrm{Ks})$

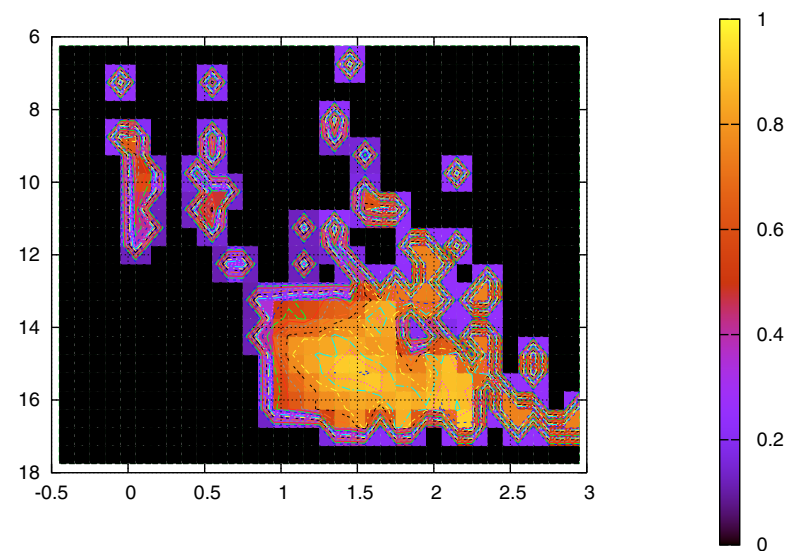

$(\mathrm{J}-\mathrm{Ks})$

Fig. 2. Top panels: number density $\left(\eta_{\mathrm{mem}}\right.$, in stars $\left.\operatorname{arcmin}^{-2}\right)$ of the probable member stars present in the decontaminated CMDs extracted within $R=10^{\prime}$ of $\mathrm{Cr} 197$ (left) and $R=15^{\prime}$ of vdB 92 (right). Bottom: survival frequency of the decontaminated stars.

and only the $\left\langle N_{\text {mem }}\right\rangle$ highest ranked stars are considered cluster members and transposed to the respective decontaminated CMD (e.g. Figs. 5 and 6).

The subtraction efficiency, i.e. the difference between the expected number of field stars (which may be fractional) and the number of stars effectively subtracted (which is integer) from each cell, summed over all cells, is $99.4 \%$ for vdB 92 and $96.7 \%$ for $\mathrm{Cr} 197$. As a caveat, the present decontamination approach implicitly assumes that the field colour-magnitude distribution somewhat matches that of the cluster. Figure 1 shows some gas and dust around both clusters, which might lead to appreciable reddening and differential reddening. While the effect in the foreground contamination may be small, it should be greater in the background. However, the 3rd Galactic quadrant location of Cr 197 and vdB 92 is expected to minimise the background contamination.

The number density of the probable member stars $\left(\eta_{\mathrm{mem}}\right)$ is shown in the $J \times\left(J-K_{\mathrm{s}}\right)$ CMDs in Fig. 2 (top panels). In both cases, most of the stars are relatively faint and red, covering a wide range in colour, which is expected of PMS stars that are somewhat affected by differential reddening. Also shown in Fig. 2 (bottom panels) is the survival frequency of the decontaminated stars. Again, the highest membership probabilities occur among the PMS stars.

\subsection{Decontaminated surface density maps}

Our decontamination approach relies upon differences in the colour and magnitude distribution of stars located in separate spatial regions. For a star cluster, which can be characterised by a single-stellar population projected against a Galactic stellar field, the decontaminated surface density is expected to present a marked excess at the assumed cluster position.

Maps of the spatial distribution of the stellar surface density $\left(\sigma\right.$, in units of stars $\operatorname{arcmin}^{-2}$ ), built with field-star decontaminated photometry to maximise the cluster/background contrast, are shown in Figs. 3 (Cr 197) and 4 (vdB 92). Also shown are the isopleths, in which cluster size and geometry can be observed. The surface density is computed in a rectangular mesh with cells $2.5^{\prime} \times 2.5^{\prime}$ wide, reaching total offsets of $|\Delta \alpha \cos (\delta)|=|\Delta \delta| \approx 45^{\prime}$ with respect to the cluster centre (Table 1).

Despite the gas and dust associated with the present clusters (Fig. 1), the central excesses show up markedly in the decontaminated surface density distributions. Cr 197 has a well-defined, approximately round, and narrow stellar distribution (Fig. 3, left panel). Its $\left(J-K_{\mathrm{S}}\right)$ colour distribution is rather uniform, within $1.0 \lesssim\left(J-K_{\mathrm{s}}\right) \lesssim 1.8$, with a blueward dip, owing to the few bright and rather blue stars, about the centre (right panel). The stellar distribution of vdB 92, on the other hand, presents a broad and elongated external region (Fig. 4, left panel) with a complex colour distribution (right panel) within $0.8 \lesssim\left(J-K_{\mathrm{s}}\right) \lesssim 2.5$. If most of these colour variations (with respect to the average) are caused by non-uniform reddening - and not by systematic differences in the stellar content - the upper limits to the differential reddening distribution would be $\Delta A_{V} \lesssim 2.5$ mag for $\mathrm{Cr} 197$ and $\Delta A_{V} \lesssim 5$ mag for vdB 92 . 

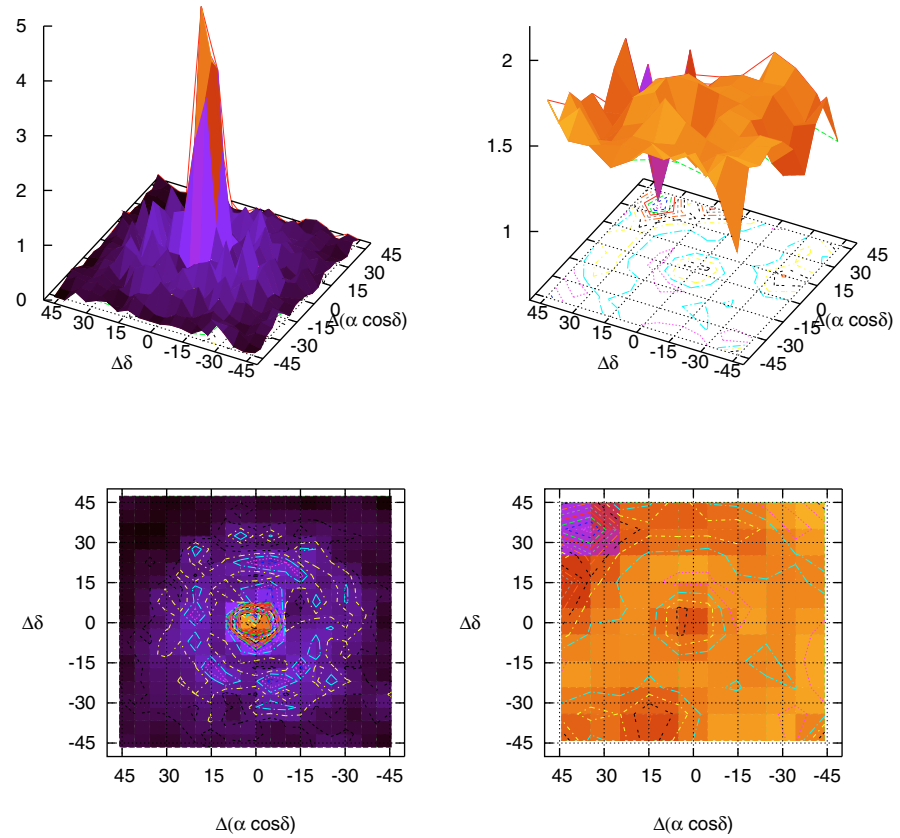

Fig. 3. Left: stellar surface density $\sigma\left(\right.$ stars $\left.\operatorname{arcmin}^{-2}\right)$ computed with field-decontaminated photometry to enhance the cluster/background contrast of Cr 197. Right: average $\left(J-K_{\mathrm{s}}\right)$ colour. $\Delta(\alpha \cos (\delta))$ and $\Delta \delta$ in arcmin.
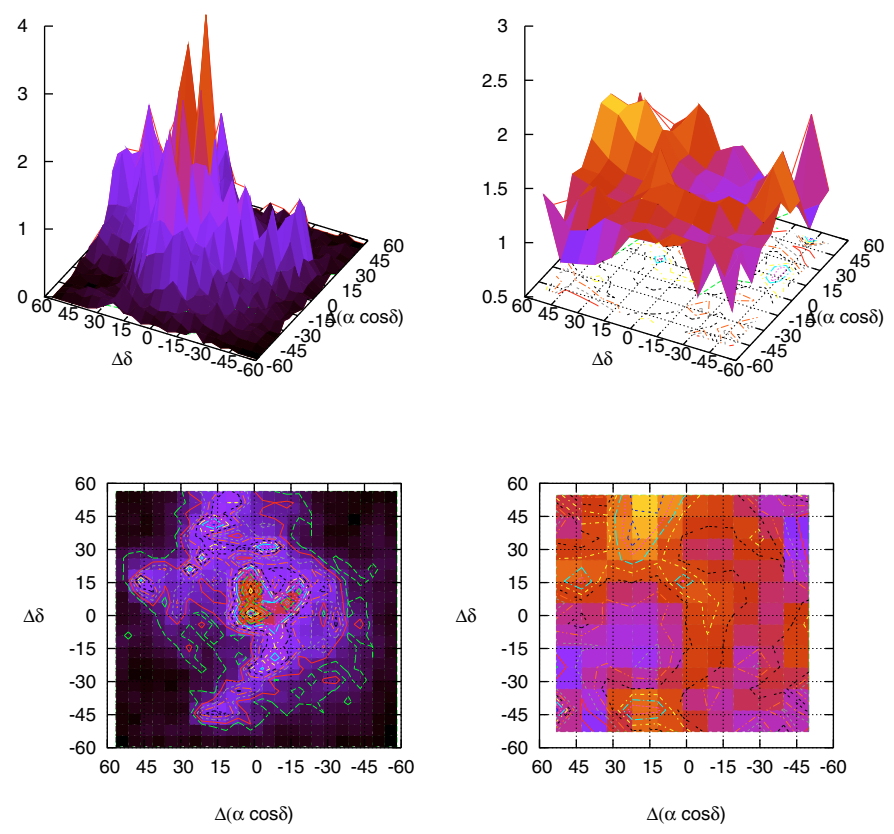

Fig. 4. Same as Fig. 3 for vdB 92.

\section{Fundamental parameters}

The top panels of Fig. 5 show $J \times\left(J-K_{\mathrm{s}}\right)$ CMDs built with the raw photometry of $\mathrm{Cr} 197$ and vdB 92. In both cases, the sampled region contains most of the cluster stars (Fig. 8). Features typical of very young OCs, such as a relatively vertical and poorlypopulated MS, together with a large population of faint and red PMS stars, can be seen when these CMDs are compared to those extracted from the equal-area offset fields ${ }^{4}$ (middle panels).

\footnotetext{
${ }^{4}$ The equal-area field extractions are only used for qualitative comparisons, since the decontamination uses a wide surrounding ring area (Sect. 3.1).
}

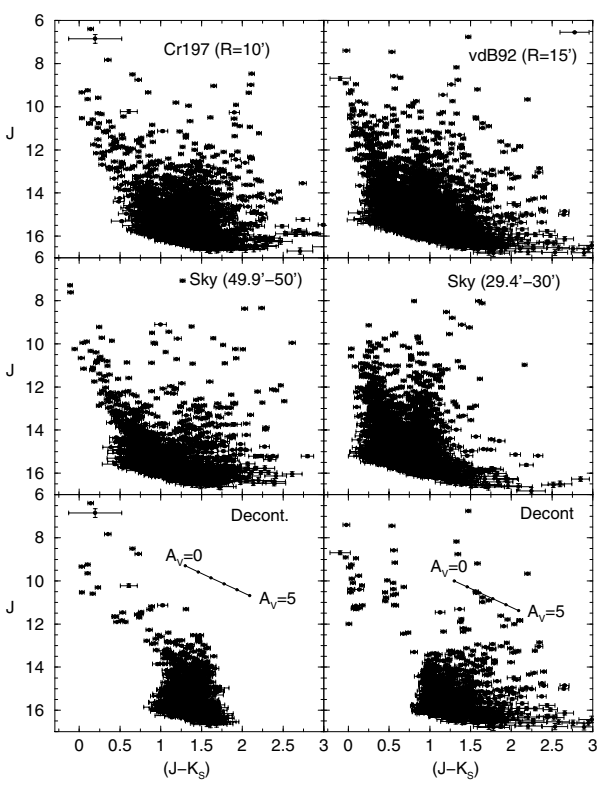

Fig. 5. $J \times\left(J-K_{\mathrm{s}}\right)$ CMDs of $\mathrm{Cr} 197$ (left) and vdB 92 (right) showing the observed photometry for representative regions (top) and the equalarea comparison fields (middle). The decontaminated CMDs are shown in the bottom panels. Reddening vectors for $A_{V}=0-5$ are shown in the bottom panels.

As expected, the decontaminated CMDs (bottom panels of Fig. 5) essentially contain the typical stellar sequences of mildly reddened young clusters, with a developing MS and a significant population of PMS stars. Also, in both cases, the colour distribution at faint magnitudes $(J \gtrsim 13)$ is wider than the spread predicted purely by PMS models (Fig. 6), which implies internal differential reddening. To examine this issue we include in Figs. 5, 6 reddening vectors computed with the 2MASS ratios (Sect. 3) for visual absorptions $A_{V}=0$ to 5. Taking the PMS isochrone fit into account (Fig. 6), the differential reddening appears to be lower than $\Delta A_{V}=5$, especially for $\mathrm{Cr} 197$, which is consistent with the values estimated in Sect. 3.2.

The fundamental parameters are derived by means of the constraints provided by the field-decontaminated CMD morphologies, especially the combined MS and PMS star distribution (Fig. 6). We adopt solar metallicity isochrones because both objects are young and located not far from the Solar circle (see below), a region essentially occupied by $[\mathrm{Fe} / \mathrm{H}] \approx 0.0 \mathrm{OCs}$ (Friel 1995). Padova isochrones (Girardi et al. 2002) computed with the 2MASS $J, H$, and $K_{\mathrm{s}}$ filters ${ }^{5}$ are used to represent the MS. Isochrones of Siess et al. (2000) with ages in the range 0.2-10 Myr are used to characterise the PMS sequences. Since the decontaminated CMD morphologies are very similar and typical of young ages (Fig. 6), a similar isochrone solution is expected to apply to both objects.

As summarised in Naylor \& Jeffries (2006), sophisticated approaches are available for analytical CMD fitting, especially the MS. However, given the poorly-populated MSs, the 2MASS photometric uncertainties for the fainter stars, the large population of PMS stars, and the differential reddening, we applied the direct comparison of isochrones with the decontaminated CMD morphology. The fits are made by eye, taking the combined MS

\footnotetext{
5 http://stev.oapd.inaf.it/cgi-bin/cmd. These isochrones are very similar to the Johnson-Kron-Cousins ones (e.g. Bessel \& Brett 1988), with differences of at most $0.01 \mathrm{mag}$ in colour (Bonatto et al. 2004).
} 


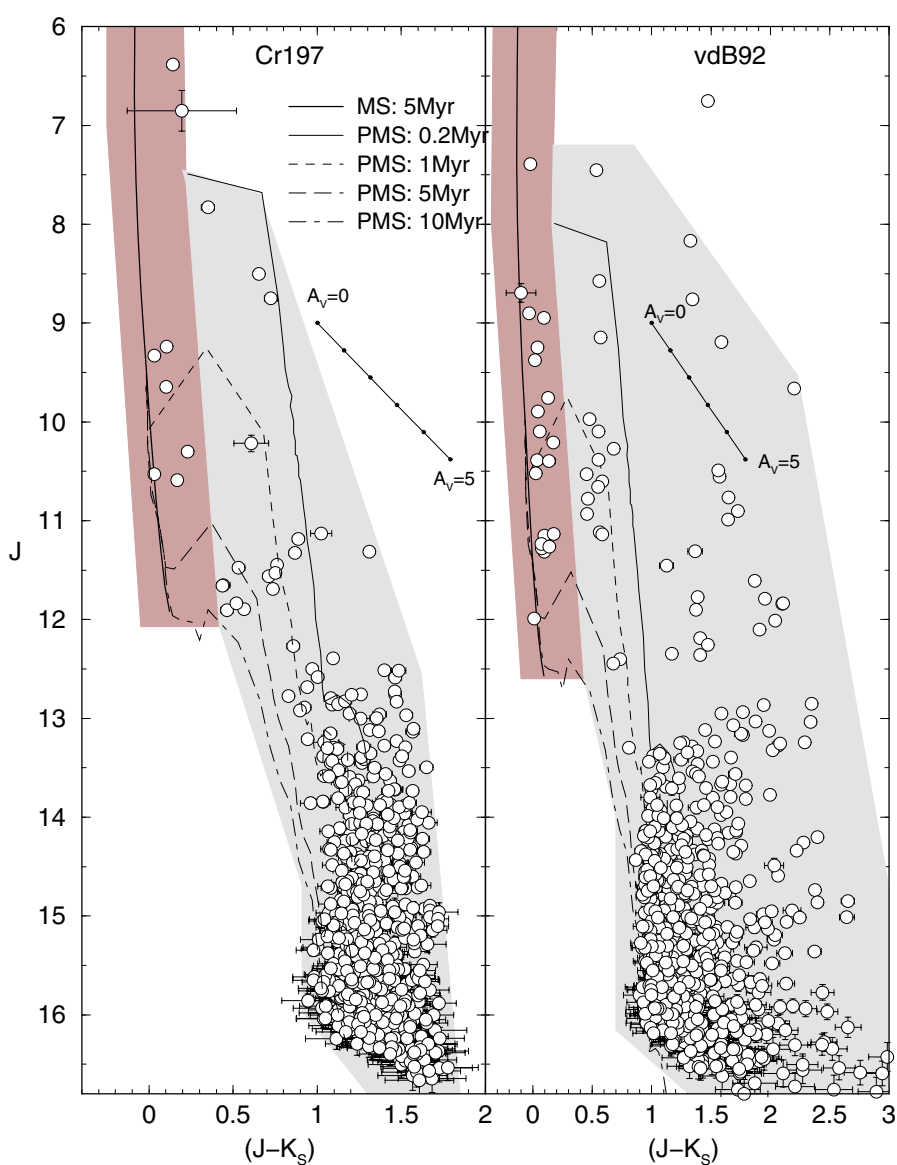

Fig. 6. Adopted MS+PMS isochrone solutions to the decontaminated CMDs. Reddening vectors as in Fig. 5. Shaded polygons show the MS (dark-grey) and PMS (light-grey) colour-magnitude filters (Sect. 5).

and PMS stellar distributions as constraints, allowing as well for variations due to photometric uncertainties (which, given the restrictions imposed in Sect. 3, are small in both cases), and differential reddening. Specifically, we start with the MS+PMS isochrones set for zero distance modulus and reddening, and apply shifts in magnitude and colour to them. We take as bestfit the apparent distance modulus and reddening that simultaneously account for the blue border of the MS and PMS stellar distributions. Both clusters present a significant fraction of stars redder than the youngest PMS isochrone. Most of this $\left(J-K_{\mathrm{s}}\right)$ excess towards the red probably stems from differential reddening. Below, we discuss the results, which are shown in Fig. 6.

Cr 197: Given the poorly-populated and nearly vertical MS, acceptable fits to the decontaminated MS are obtained with any isochrone with age in the range 1-10 Myr. If we consider the differential reddening, this age spread is consistent with the PMS stars, which are contained within the 0.2 Myr and $10 \mathrm{Myr}$ isochrones as well. Thus, we take the $5 \mathrm{Myr}$ isochrone as a representative solution, and allow for a \pm 4 Myr age spread in the star formation.

With the adopted solution, the fundamental parameters of $\mathrm{Cr} 197$ are the near-IR reddening $E(J-H)=0.10 \pm 0.05$ $\left(E(B-V)=0.34 \pm 0.16\right.$ or $\left.A_{V}=1.0 \pm 0.5\right)$, the observed and absolute distance moduli $(m-M)_{J}=10.4 \pm 0.4$ and $(m-M)_{\mathrm{O}}=10.11 \pm 0.42$, respectively, and the distance from the Sun $d_{\odot}=1.05 \pm 0.20 \mathrm{kpc}$. This distance is compatible with what is obtained for the B4 II star HIP 42908 ( $B=7.65$ and $V=7.32), d_{\odot}=1.1 \mathrm{kpc}$. With $R_{\odot}=7.2 \pm 0.3 \mathrm{kpc}$

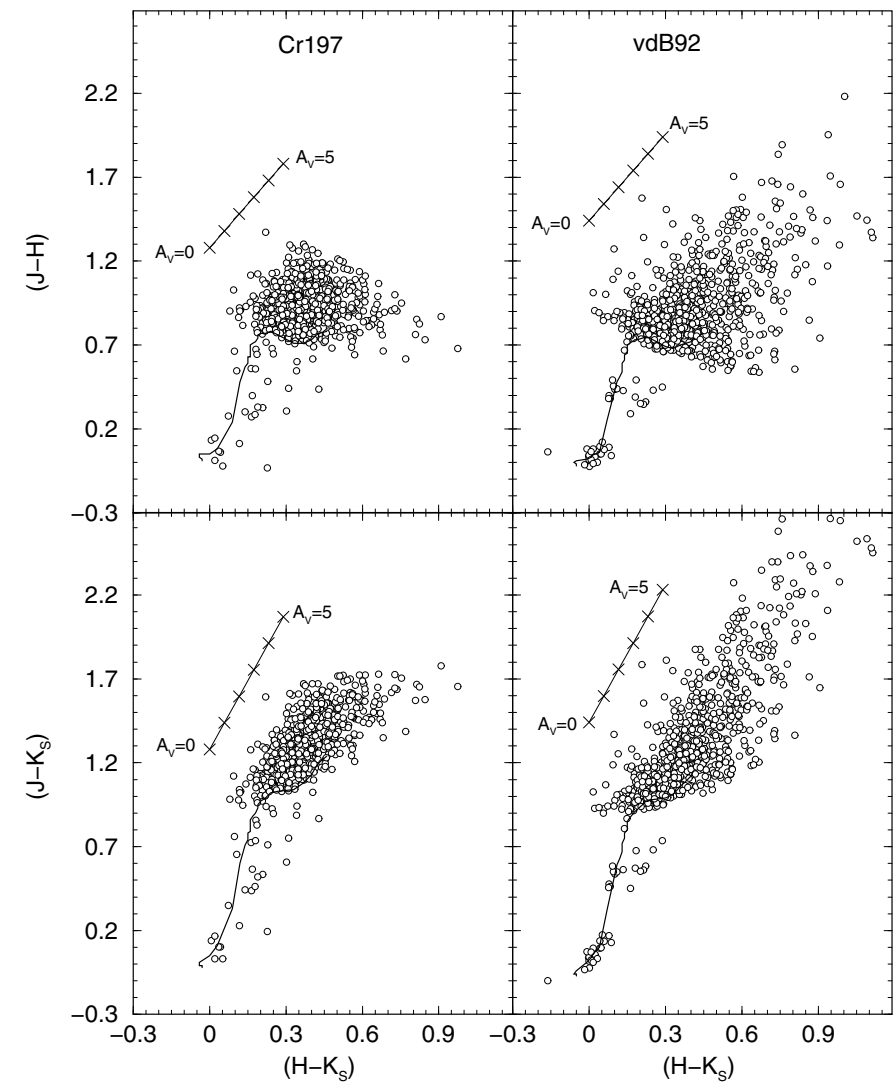

Fig. 7. Colour-colour diagrams showing the decontaminated photometry and the 5 Myr PMS track (Siess et al. 2000), set with the derived reddening values (Sect. 4). MS stars lie on the blue side of the diagram. Error bars have been omitted for clarity. Reddening vectors as in Figs. 5 and 6.

(Bica et al. 2006) as the Sun's distance to the Galactic centre ${ }^{6}$, the Galactocentric distance of $\mathrm{Cr} 197$ is $R_{\mathrm{GC}}=7.5 \pm 0.1 \mathrm{kpc}$, which puts it $\approx 0.3 \mathrm{kpc}$ outside the Solar circle. This solution is shown in Fig. 6. The projected coordinate components are $\left(x_{\mathrm{GC}}, y_{\mathrm{GC}}, z_{\mathrm{GC}}\right) \approx(-7.4,-1.4,0.0)$ in $\mathrm{kpc}$, which put $\mathrm{Cr} 197$ at the Orion-Cygnus and Sagittarius-Carina interarm region (e.g. Momany et al. 2006). We also computed the integrated apparent and absolute magnitudes in the 2MASS bands for the member stars within $R \leq R_{\mathrm{RDP}}$, together with the reddening corrected colours. The results are $m_{J}=5.64 \pm 0.07, M_{J}=-4.76 \pm 0.12$, $(J-H)=0.19 \pm 0.09$, and $\left(J-K_{\mathrm{s}}\right)=0.32 \pm 0.09$.

vdB 92 : Similar to Cr 197, any isochrone with age within 1-10 Myr provides an acceptable fit to the nearly vertical MS, while the PMS distribution is essentially contained within the 0.2-10 Myr isochrones. With this solution, the fundamental parameters of vdB 92 are $E(J-H)=0.07 \pm 0.04(E(B-V)=$ $0.22 \pm 0.13$ or $\left.A_{V}=0.7 \pm 0.4\right),(m-M)_{J}=10.9 \pm 0.4$ $(m-M)_{\mathrm{O}}=10.71 \pm 0.41, d_{\odot}=1.38 \pm 0.26 \mathrm{kpc}$, and $R_{\mathrm{GC}}=$ $8.3 \pm 0.2 \mathrm{kpc}$, thus $\approx 1.0 \mathrm{kpc}$ outside the Solar circle. Thus, with the coordinate components $\left(x_{\mathrm{GC}}, y_{\mathrm{GC}}, z_{\mathrm{GC}}\right) \approx(-8.2,-1.0,-0.1)$, vdB 92 approximately coincides with the Orion-Cygnus arm (e.g. Momany et al. 2006). SIMBAD provides 5 stars with spectral type and optical photometry to within $\approx 10^{\prime}$ of the central coordinates of vdB 92 (Table 1). These are HRW 14 (B7 III,

6 Derived by means of the globular cluster spatial distribution. Recently, Trippe et al. (2008) have found $R_{\mathrm{GC}}=8.07 \pm 0.32 \mathrm{kpc}$, while Ghez et al. (2008) find $R_{\mathrm{GC}}=8.0 \pm 0.6 \mathrm{kpc}$ or $R_{\mathrm{GC}}=8.4 \pm 0.4 \mathrm{kpc}$, under different assumptions. 
C. Bonatto and E. Bica: Collinder 197 and vdB 92: dissolving star clusters?

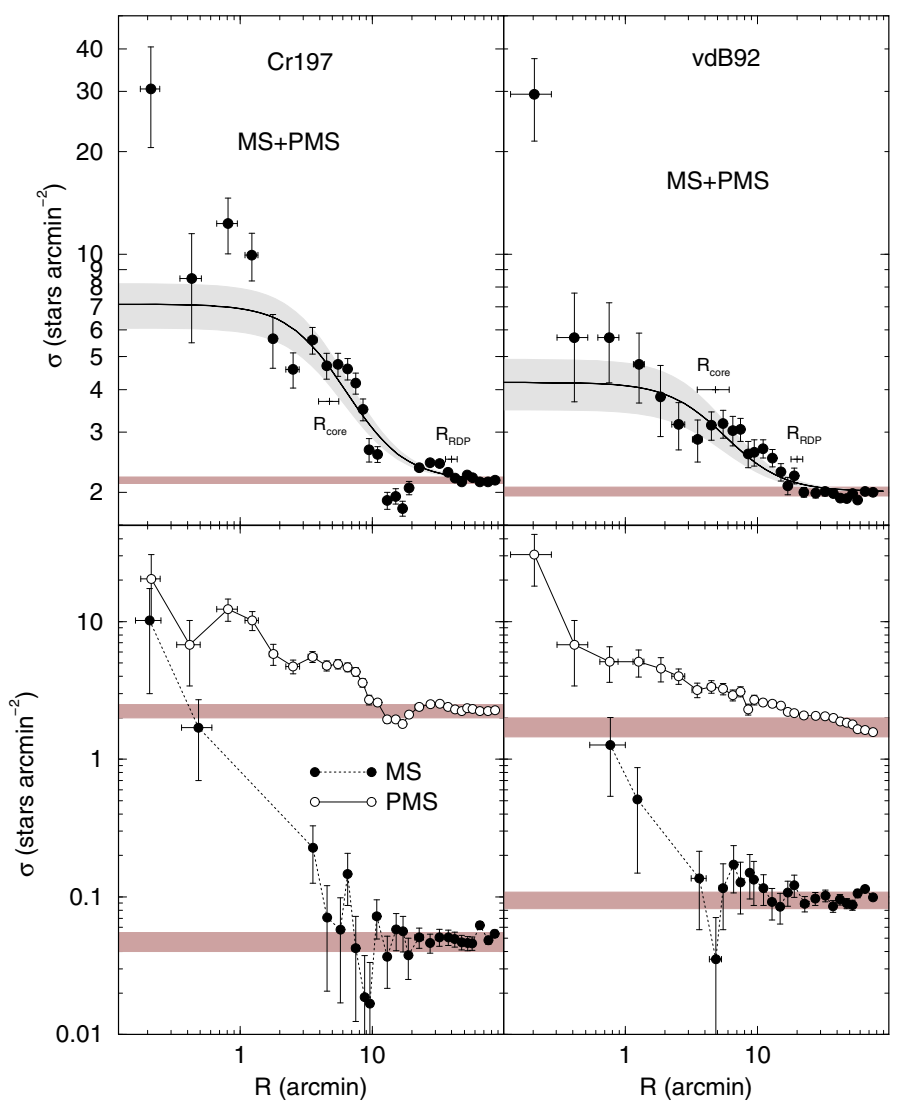

Fig. 8. Top: stellar RDPs built for the MS and PMS stars combined, together with the best-fit King-like profile (solid line), the $1 \sigma$ uncertainty (light-shaded region), and the background level (shaded polygon). Bottom: RDPs built isolately for the MS and PMS stars.

$B=12.67$ and $V=12.09), \mathrm{BD}-111763(\mathrm{~B} 1.5 \mathrm{~V}, B=8.90$ and $V=8.91), \mathrm{BD}-111761(\mathrm{~B} 2 \mathrm{~V}, B=9.32$ and $V=9.25)$, HIP 34133 (B0 V, $B=7.31$ and $V=7.34$ ), and NSV 3364 (B3 V, $B=9.55$ and $V=9.41)$. The average spectroscopic distance of these stars is $d_{\odot}=1.4 \pm 0.2 \mathrm{kpc}$, in excellent agreement with the photometric value for $\operatorname{vdB} 92$. For vdB 92 we derive $m_{J}=6.35 \pm 0.03, M_{J}=-4.53 \pm 0.41,(J-H)=0.54 \pm 0.05$, and $\left(J-K_{\mathrm{s}}\right)=1.01 \pm 0.04$. Thus, $\mathrm{Cr} 197$ is intrinsically brighter and somewhat bluer than vdB 92.

Besides the similar MS age, Cr 197 and vdB 92 share a significant age spread ( $10 \mathrm{Myr})$ implied by the PMS stars, which indicates a non-instantaneous star formation. We found a similar scenario in our previous studies of, e.g. NGC 4755, NGC 6611, and NGC 2244.

Although with a relatively low foreground absorption $\left(A_{V} \lesssim\right.$ 1 ), both cases present a significant fraction of PMS stars redder (and fainter) than predicted by the PMS tracks (Fig. 6). Indeed, when transposed to near-IR colour-colour diagrams (Fig. 8), the age and reddening solutions derived for $\mathrm{Cr} 197$ and vdB 92 consistently match most of their field-star decontaminated photometry, but a significant fraction of the PMS stars appear to be very reddened. Most of the very red PMS stars occur along the respective reddening vectors. However, a small fraction appear to present an abnormal excess in $\left(H-K_{\mathrm{s}}\right)$, especially vdB 92 , in the $(J-H) \times\left(H-K_{\mathrm{s}}\right)$ diagram, which may come from PMS stars still bearing circumstellar discs, hence with excess in $H$ (e.g. Furlan et al. 2009; Bonatto et al. 2006a).

\section{Structural parameters}

We derive structural parameters by means of the RDPs, which are the projected stellar number density around the cluster centre. Noise in the RDPs is minimised with colour-magnitude filters (Fig. 6), which exclude stars with colours incompatible with those of the cluster ${ }^{7}$. In previous works we have shown that this filtering procedure enhances the RDP contrast relative to the background, especially in crowded fields (e.g. Bonatto \& Bica 2007).

Rings of increasing width with distance from the cluster centre are used to preserve spatial resolution over the full radial range. The set of ring widths used is $\Delta R=$ $0.25,0.5,1.0,2.5$, and $5^{\prime}$, respectively for $0^{\prime} \leq R<0.5^{\prime}$, $0.5^{\prime} \leq R<2^{\prime}, 2^{\prime} \leq R<5^{\prime}, 5^{\prime} \leq R<20^{\prime}$, and $R \geq 20^{\prime}$. The residual background level of each RDP is the average number density of field stars. The $R$ coordinate (and uncertainty) of each ring corresponds to the average position and standard deviation of the stars inside the ring. The resulting RDPs are shown in Fig. 8. As a caveat we note that the projected stellar distribution of vdB 92 is not smooth and radially symmetric, especially at the outskirts (Fig. 4). Thus, the use of circular rings to build the RDP might introduce some noise at large radii, but with little effect on the central parts.

We fit the RDPs with the function $\sigma(R)=\sigma_{\mathrm{bg}}+\sigma_{0} /(1+$ $\left.\left(R / R_{\mathrm{c}}\right)^{2}\right)$, where $\sigma_{0}$ and $\sigma_{\mathrm{bg}}$ are the central and residual background stellar densities, and $R_{\mathrm{c}}$ is the core radius. Applied to star counts, this function is similar to the one used by King (1962) for the surface-brightness profiles in the central parts of globular clusters ${ }^{8}$. To minimise degrees of freedom, only $\sigma_{0}$ and $R_{\mathrm{c}}$ are derived from the fit, while $\sigma_{\mathrm{bg}}$ is previously measured in the surrounding field and kept fixed.

The best-fit solutions (and uncertainties) are shown in Fig. 8, and the corresponding structural parameters are given in Table 2. In addition, we estimate the cluster radius $\left(R_{\mathrm{RDP}}\right)$, i.e., the distance from the centre where the cluster RDP and field fluctuations are statistically indistinguishable (e.g. Bonatto \& Bica 2005), and the density contrast parameter $\delta_{\mathrm{c}}=1+\sigma_{0} / \sigma_{\mathrm{bg}}$ (Table 2). $R_{\mathrm{RDP}}$ may be taken as an observational truncation radius, whose value depends both on the radial distribution of member stars and the field density.

Within uncertainties, the adopted King-like function provides a reasonable description of the RDPs for $R \gtrsim 1^{\prime}$ (Fig. 8). The drop in the RDP of CR 197 for $13 \lesssim R\left(^{\prime}\right) \lesssim 18$ is probably related to enhanced dust absorption. However, the RDP measured in the innermost region $\left(R \lesssim 0.3^{\prime}\right)$ in both cases presents a significant $(\gtrsim 3 \sigma)$ excess, or cusp, over the fit. In old star clusters, this feature has been attributed to a post-core collapse structure, as detected in some globular clusters (e.g. Trager et al. 1995). Some Gyr-old OCs, e.g. NGC 3960 (Bonatto \& Bica 2006) and LK 10 (Bonatto \& Bica 2009a), also display such a dynamical evolution-related feature. Interestingly, this cusp also occurs in the RDP of some very young ( $\$ 10 \mathrm{Myr})$ clusters, e.g. NGC 2244 (Bonatto \& Bica 2009b), NGC 6823 (Bica et al. 2008a), Pismis 5, and NGC 1931 (Bonatto \& Bica 2009c). Such time scales are too short for clusters to evolve into a post-core collapse. Thus, the cusp in young clusters is probably related to

\footnotetext{
7 They are wide enough to include cluster MS and PMS stars, together with the photometric uncertainties and binaries (and other multiple systems).

${ }^{8}$ Because of the relatively low number of stars in the present OCs, fluctuations in surface-brightness profiles are expected to be higher than those in RDPs. Alternative RDP fit functions are discussed in Bonatto \& Bica (2008).
} 
molecular cloud fragmentation and/or star formation, and may suggest important early deviation from dynamical equilibrium (Sect. 8). As a dynamically-related alternative explanation for the RDP cusps, we note that numerical simulations of clusters that form dynamically cool (subvirial) and with a fractal structure (Allison et al. 2009) suggest that mass segregation may occur on time scales comparable to their crossing times, typically a few Myr. Also, clusters that form with significant substructure will probably develop an irregular central region, unless such a region collapses and smooths the initial substructure out.

Finally, we also show in Fig. 8 (bottom panels) the RDPs built after isolating the MS and PMS stars by means of the respective colour-magnitude filters (Fig. 6). In both clusters, MS stars are more concentrated than the PMS ones and, as expected, the fraction of PMS stars (with respect to the total number) increases with cluster radius, and largely dominates the MS stars, especially for $R \gtrsim 5^{\prime}$. The innermost region of $\mathrm{vdB} 92$ is primarily occupied by PMS stars. The different residual background levels between the PMS and MS RDPs reflect the dominant presence of faint and red stars in the field, with respect to the bright and blue ones. As a consequence, the density-contrast parameter of the MS stars in Cr 197 is $\approx 20$ higher than that of the PMS stars. The absence of MS stars in the innermost RDP bin precludes this comparison for vdB 92.

Considering the fit parameters (Table 2), the density-contrast parameter is relatively low in both cases, $2.1 \lessgtr \delta_{\mathrm{c}} \lesssim 3.3$. However, when the measured innermost RDP value is considered (Fig. 8), it increases to $\delta_{\mathrm{c}}=15.1 \pm 4.6$ and $\delta_{\mathrm{c}}=15.7 \pm 4.0$, respectively, for $\mathrm{Cr} 197$ and $\mathrm{vdB} 92$. The latter values are more representative of the visual contrast produced by the few relatively bright stars in the central regions of both objects.

Alternatively, we fitted the RDPs allowing for variations of the background level. Within the uncertainties, the corresponding parameters (Table 2) are compatible with the previous (fixed background level) ones. Taken at face value, the core radii derived from the King-like fits - of $\operatorname{Cr} 197\left(R_{\mathrm{c}} \approx 1.5 \mathrm{pc}\right)$ and $\operatorname{vdB} 92\left(R_{\mathrm{c}} \approx 2.0 \mathrm{pc}\right)$ are somewhat larger than the median value of the $R_{\mathrm{c}}$ distribution derived for a sample of relatively nearby OCs by Piskunov et al. (2007).

\section{Stellar mass estimate}

By far, the CMDs of Cr 197 and vdB 92 are dominated in number by PMS stars, followed by the developing, poorly-populated MS (Fig. 6), which implies that most of the cluster mass is stored in the PMS stars. To compute the cluster masses we work with the field-decontaminated photometry within $R \leq R_{\mathrm{RDP}}$ and count the number of stars that belong either to the MS or PMS.

For the MS, the corresponding mass of each star is taken from the mass-luminosity relation derived from the isochrone solution (Sect. 4). The MS mass range, in both cases, lies within $\approx 2-30 M_{\odot}$. Summing up these values for all stars produces the total number $\left(n_{\mathrm{MS}}\right)$ and mass $\left(m_{\mathrm{MS}}\right)$ of MS stars. Because of the differential reddening, individual masses cannot be assigned to the PMS stars. Thus, we simply count the number of PMS stars and adopt an average mass value to estimate $n_{\text {PMS }}$ and $m_{\text {PMS }}$. To compute the average PMS mass value we use the Kroupa (2001) initial mass function ${ }^{9}$ for PMS masses between $0.08-7 M_{\odot}$. The result is $\bar{m}_{\mathrm{PMS}}=0.6 M_{\odot}$. Taking the uncertainty in $R_{\mathrm{RDP}}$ into account (Table 2), we obtain for Cr $197 n_{\mathrm{MS}}=26_{-7}^{+10}$,

\footnotetext{
9 Defined as $\mathrm{d} N / \mathrm{d} M \propto m^{-(1+\chi)}$, it assumes the slopes $\chi=0.3 \pm 0.5$ for $0.08 \leq m\left(M_{\odot}\right) \leq 0.5, \chi=1.3 \pm 0.3$ for $0.5 \leq m\left(M_{\odot}\right) \leq 1.0$, and $\chi=1.35$ for $m\left(M_{\odot}\right)>1.0$.
}

Table 3. Gaussian fit parameters.

\begin{tabular}{cccccc}
\hline \hline Cluster & $\overline{\mu_{\alpha} \cos (\delta)}$ & $\sigma_{\alpha}$ & $\overline{\mu_{\delta}}$ & $\sigma_{\delta}$ & $\begin{array}{c}\sigma_{\mathrm{v}} \\
\left(\mathrm{km} \mathrm{s}^{-1}\right)\end{array}$ \\
$\left(\mathrm{km} \mathrm{s}^{-1}\right)$ & $\left(\mathrm{km} \mathrm{s}^{-1}\right)$ & $\left(\mathrm{km} \mathrm{s}^{-1}\right)$ & $\left(\mathrm{km} \mathrm{s}^{-1}\right)$ \\
\hline $\mathrm{Cr} 197$ & $-22.2 \pm 4.1$ & $10.2 \pm 3.7$ & $-8.1 \pm 4.0$ & $14.4 \pm 4.3$ & $22 \pm 5$ \\
$\mathrm{Cr} 197$ & $-48.5 \pm 5.0$ & $6.6 \pm 3.6$ & $+30.6 \pm 2.1$ & $9.9 \pm 1.8$ & $15 \pm 3$ \\
vdB 92 & $-18.0 \pm 1.6$ & $11.1 \pm 1.3$ & $+14.5 \pm 2.1$ & $13.1 \pm 1.7$ & $21 \pm 2$ \\
\hline
\end{tabular}

Notes. $\sigma_{\alpha}$ and $\sigma_{\delta}$ are the velocity dispersions in right ascension and declination, respectively. Assuming spherical symmetry, we define $\sigma_{\mathrm{v}}^{2}=$ $\frac{3}{2}\left(\sigma_{\alpha}^{2}+\sigma_{\delta}^{2}\right)$.

$m_{\mathrm{MS}}=172_{-46}^{+66} M_{\odot}, n_{\mathrm{PMS}}=809_{-62}^{+170}$, and $m_{\mathrm{PMS}}=485_{-37}^{+78} M_{\odot}$. Thus, Cr 197 contains $\approx 835$ stars and $M_{\text {clu }} \approx 660_{-59}^{+102} M_{\odot}$ of stellar mass. The values for vdB 92 are $n_{\mathrm{MS}}=13_{-5}^{+8}, m_{\mathrm{MS}}=$ $106_{-41}^{+65} M_{\odot}, n_{\mathrm{PMS}}=1067_{-50}^{+130}$, and $m_{\mathrm{PMS}}=640_{-30}^{+78} M_{\odot}$. The cluster vdB 92 thus contains $\approx 1080$ stars and $M_{\mathrm{clu}} \approx 750_{-51}^{+101} M_{\odot}$ of stellar mass.

Both objects present similar values of member MS and PMS stars and total mass. Besides, as anticipated by the CMDs (Fig. 6), the MS is poorly-populated in each case, to the point where the mass stored in the PMS stars is the dominant component of the cluster mass (74\% in $\mathrm{Cr} 197$ and 85\% in vdB 92). However, these masses may still be somewhat higher, since because of the differential reddening and the 2MASS photometric limit, we may not detect the very low-mass PMS stars. Also, given the presence of some dust and gas in Cr 197 and vdB 92 (Fig. 1), their total cluster masses may be a bit higher than the present estimates. Finally, that most of the mass is stored in the faint PMS stars may explain the significant discrepancies with respect to previous mass determinations made in the optical (Sect. 2).

\section{Proper motions}

Given the relative proximity of both clusters (Table 1), we can use proper motion data to probe kinematical properties of the member stars. For this purpose we use the Third US Naval Observatory CCD Astrograph Catalog (UCAC3, Zacharias et al. 2010), which also provides the 2MASS photometry for each star.

Proper motions were obtained for the same central coordinates and extraction radius as those used to extract the 2MASS photometry (Table 1). The region analysed is contained within $R=10^{\prime}$, both for Cr 197 and vdB 92, with the comparison field located within $60^{\prime}-80^{\prime}$. Also, we applied the respective colourmagnitude filters (Sect. 5) before computing the proper motion distributions. Conversion from mas $\mathrm{yr}^{-1}$ to $\mathrm{km} \mathrm{s}^{-1}$ was based on the respective cluster distances (Table 1).

The proper motion distributions $(\phi(\mu)=\mathrm{d} N / \mathrm{d} \mu)$ in units of number of stars per $\operatorname{arcmin}^{2}$ per $\mathrm{km} \mathrm{s}^{-1}$, for both the $R=10^{\prime}$ and field regions, are shown in Fig. 9. The intrinsic distribution corresponds to the subtraction of the field contribution to the $R=10^{\prime}$ region.

Cr 197 appears to require two components of similar velocity dispersion to describe its intrinsic proper motion distribution, while a single one applies to vdB 92 . The best-fits, obtained with Gaussians, are shown in Fig. 9, and the corresponding parameters are given in Table 3 . In velocity space, the 2 proper motion distributions of $\mathrm{Cr} 197$ have peaks separated by $\approx 26 \mathrm{~km} \mathrm{~s}^{-1}$ and $\approx 39 \mathrm{~km} \mathrm{~s}^{-1}$, respectively in right ascension and declination. 
C. Bonatto and E. Bica: Collinder 197 and vdB 92: dissolving star clusters?

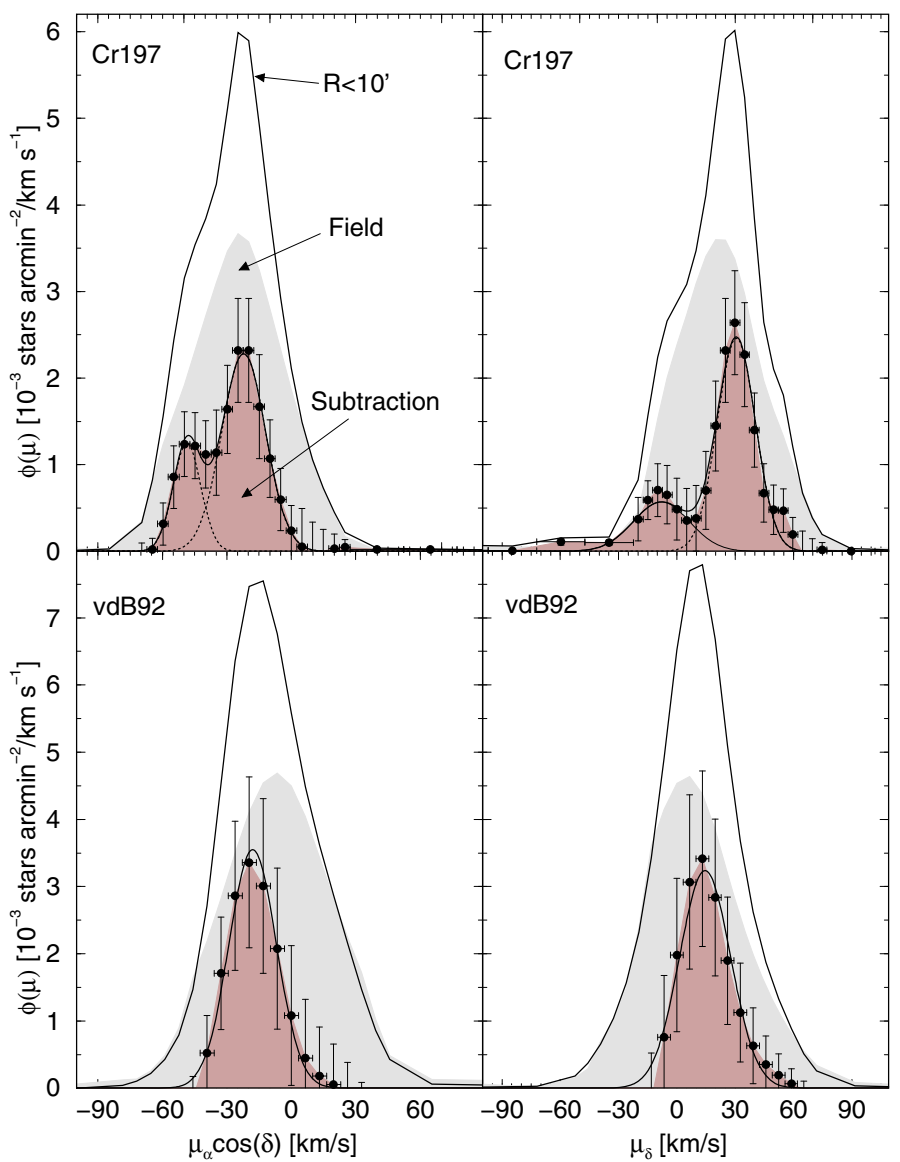

Fig. 9. Proper motion distribution of $\mathrm{Cr} 197$ (top) and vdB 92 (bottom). In both cases, the field contribution (light-grey) is subtracted from that of the $R<10^{\prime}$ region (heavy-solid line) to produce the intrinsic distribution (heavy-grey). Gaussians are fitted to the resulting distributions (see text for details). For clarity, error bars are only shown in the subtracted profiles.

The spatial velocity dispersion of a cluster that is in virial equilibrium can be expressed as (Spitzer 1987) $\sigma^{2}=\frac{G M_{\mathrm{D}}}{\eta R_{\mathrm{eff}}} \approx$ $0.5\left(\frac{M_{\mathrm{D}}}{10^{3} M_{\odot}}\right)\left(\frac{R_{\mathrm{eff}}}{1 \mathrm{pc}}\right)^{-1}\left(\mathrm{~km} \mathrm{~s}^{-1}\right)^{2}$, where $G$ is the gravitational constant, $M_{\mathrm{D}}$ the dynamical mass (assumed to be stored only in stars), $\eta \approx 9.75$ is a constant (that roughly depends on the density profile), and $R_{\text {eff }}$ is the effective, or half-light, radius. Thus, clusters with $M_{\mathrm{D}} \sim 10^{3} M_{\odot}$ and $R_{\text {eff }} \sim 1 \mathrm{pc}$ are expected to present $\sigma \sim 1 \mathrm{~km} \mathrm{~s}^{-1}$.

In the last column of Table 3, we provide an estimate of the spatial velocity dispersion $\left(\sigma_{\mathrm{v}}\right)$, computed by assuming spherical symmetry for $\mathrm{Cr} 197$ and vdB 92, $\sigma_{\mathrm{v}}^{2}=\frac{3}{2}\left(\sigma_{\alpha}^{2}+\sigma_{\delta}^{2}\right)$. Clearly, both clusters present $\sigma_{\mathrm{v}}$ values that are much higher than the expected dynamical ones ${ }^{10}$. Such a super-virial state has been linked to the impulsive expulsion of the parental molecular cloud's residual gas, driven essentially by the strong winds of massive stars and supernovae (Goodwin \& Bastian 2006), which leads to the high rate of dissolution of the young clusters (e.g. Lada \& Lada 2003). A similar scenario has been observed to occur in the dissolving OC NGC 2244, which has a spatial velocity dispersion of $\sigma_{\mathrm{v}} \approx 35 \mathrm{~km} \mathrm{~s}^{-1}$ (Chen et al. 2004), which by far exceeds the expected dynamical value (de Grijs et al. 2008).

\footnotetext{
${ }^{10}$ Because the velocity dispersions considered here are derived from proper motions, the binaries' contribution to $\sigma_{\mathrm{v}}$ (Gieles et al. 2008) is expected to be minimal.
}
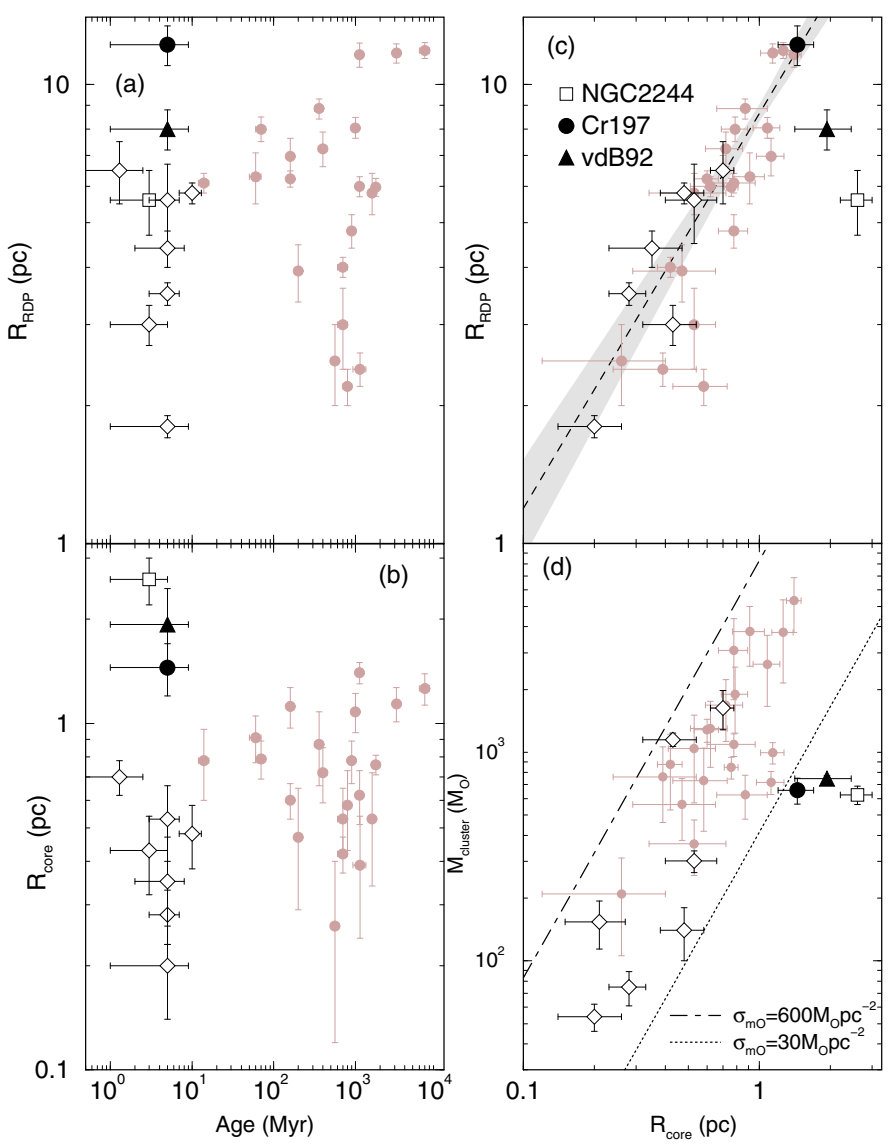

Fig. 10. Diagrams dealing with astrophysical parameters of OCs. Greyshaded circles: template OCs. The young OC NGC 2244 is indicated for comparison purposes. The analytical relations in panels c) and d) are discussed in the text.

\section{Discussion}

In previous sections we derived a set of astrophysical parameters for $\mathrm{Cr} 197$ and vdB 92. We now compare them with the same parameters derived (using the same methods and photometry) for a sample of classical OCs, basically gravitationally bound clusters, characterised by a range of properties (e.g. age, mass, size, etc.) and located in different environments. The template sample contains some relatively nearby and bright OCs (Bonatto \& Bica 2005; Bonatto et al. 2006a) together with a few projected towards the central Galaxy (Bonatto \& Bica 2007). The young OCs NGC 6611 (Bonatto et al. 2006b), NGC 6823 (Bica et al. 2008a) and NGC 2239 (Bonatto \& Bica 2009b), are included for comparison with gravitationally bound objects of similar ages, while the young NGC 2244 (Bonatto \& Bica 2009b) might be dynamically evolving into an $\mathrm{OB}$ association.

The comparisons are based on diagnostic-diagrams built with template clusters (Fig. 10). Panels (a) and (b) deal with the dependence of cluster $\left(R_{\mathrm{RDP}}\right)$ and core $\left(R_{\mathrm{c}}\right)$ radii on age, respectively. In terms of $R_{\mathrm{RDP}}$, vdB 92 and, especially, Cr 197 present higher values than the other young OCs in the sample. Cr 197 is as big as some Gyr-old OCs. With respect to $R_{\mathrm{c}}$, both objects have a significantly larger core than the classical (young and old) OCs, comparable to the core of the disrupting NGC 2244. Within uncertainties, Cr 197 follows the relation $R_{\mathrm{RDP}}=(8.9 \pm 0.3) \times R_{\mathrm{C}}^{(1.0 \pm 0.1)}$ (panel c), derived with the template sample. Similar to NGC 2244, vdB 92 is an outlier. 
For gravitationally bound star clusters in which the projected mass density follows a King-like profile (e.g. Bonatto \& Bica 2008) characterised by a central surface mass density $\sigma_{M 0}$, the mass inside $R_{\mathrm{RDP}}$ can be expressed as a function of $R_{\mathrm{c}}, \sigma_{M 0}$, and the ratio $R_{\mathrm{RDP}} / R_{\mathrm{c}}$, as $M_{\text {clus }}=\pi R_{\mathrm{C}}^{2} \sigma_{M 0} \ln \left[1+\left(R_{\mathrm{RDP}} / R_{\mathrm{c}}\right)^{2}\right]$. With the relation between $R_{\mathrm{c}}$ and $R_{\mathrm{RDP}}$ implied by panel (c), this equation becomes $M_{\text {clus }} \approx 13.8 \sigma_{M 0} R_{\mathrm{C}}^{2}$. Thus, the template OCs are constrained within King-like distributions with $30 \lesssim \sigma_{M 0}\left(M_{\odot} \mathrm{pc}^{-2}\right) \lesssim 600$ (panel d). Cr 197 and vdB 92 (together with NGC 2244) are outliers with respect to the distribution of bound OCs in the plane $R_{\mathrm{C}} \times M_{\text {clus }}$, in the sense that their locii imply exceedingly low central mass densities $\left(4 \lesssim \sigma_{M 0}\left(M_{\odot} \mathrm{pc}^{-2}\right) \lesssim 30\right)$. Significantly smaller core radii and/or higher cluster mass would be required for them to be contained within the classical OC boundaries.

Together with the kinematical analysis (Sect. 7), a possible conclusion that can be drawn from this comparison with classical bound OCs is that $\mathrm{Cr} 197$ and vdB 92 may be evolving into OB associations and/or towards dissolution. Given their young ages, the determining factor for this behaviour is certainly related to another mechanism than age-dependent dynamical evolution. Instead, it is probably associated with the primordial star formation process, or even earlier, to the molecular cloud fragmentation. Both clusters may have been formed with dynamically hot stellar components, so are unstable against dissolution (e.g. Goodwin 2009). A similar conclusion was reached for some equally young objects, but with very different masses: NGC 2244 (Bonatto \& Bica 2009b) and Bochum 1 (Bica et al. 2008a) have comparable stellar masses $\left(M_{\text {clu }} \sim 600 M_{\odot}\right)$, while NGC 1931 and Pismis 5 (Bonatto \& Bica 2009c) are at the lowmass end $\left(M_{\text {clu }} \sim 180 M_{\odot}\right.$, and $M_{\text {clu }} \sim 60 M_{\odot}$, respectively). Thus, early star cluster dissolution appears to leave detectable imprints also on the structure (e.g. RDP) of clusters as massive as several hundred solar masses.

\section{Summary and conclusions}

Given the complex interplay among environmental conditions, star-formation efficiency, initial dynamical state of the stellar content, and the total mass converted into stars or expelled, the majority of the embedded clusters do not survive the first few ten Myr, especially the low-mass ones. In this context, it is important to investigate the structural and photometric properties of OCs (with a mass range and in different environments) that are undergoing this early phase.

In the present paper we derive astrophysical parameters and investigate the nature of the young OCs Cr 197 and vdB 92. Their location in the 3rd Galactic quadrant minimises contamination by field stars. We work with field-star decontaminated 2MASS photometry (with errors $\lesssim 0.1 \mathrm{mag}$ ), which enhances CMD evolutionary sequences and stellar RDPs, yielding more constrained fundamental and structural parameters.

The decontaminated CMDs are characterised by similar properties, an under populated and developing MS, a dominant fraction $(\gtrsim 75 \%)$ of PMS stars, and some differential reddening. In both cases, the MS and PMS CMD morphologies consistently imply a time-spread of $\sim 10 \mathrm{Myr}$ in the star formation. Thus, we set the age of Cr 197 and vdB 92 as constrained within $5 \pm 4$ Myr. Their MS+PMS stellar masses are $\approx 660_{-59}^{+102} M_{\odot}(\mathrm{Cr} 197)$ and $\approx 750_{-51}^{+101} M_{\odot}$ (vdB 92). By means of the proper motion of the member MS and PMS stars, we estimate their velocity dispersions to be in the range $\approx 15 \mathrm{~km} \mathrm{~s}^{-1}$ to $\approx 22 \mathrm{~km} \mathrm{~s}^{-1}$.
Compared to a set of classical bound OCs, both $\mathrm{Cr} 197$ and vdB 92 appear to have core and cluster radii that are abnormally large, with $R_{\mathrm{c}} \approx 1.5,1.9 \mathrm{pc}$ and $R_{\mathrm{RDP}} \approx 12,8 \mathrm{pc}$, respectively for Cr 197 and vdB 92. Structurally, the stellar RDPs follow a cluster-like profile for most of the radial range, except in the central region, where they have a pronounced cusp. At less than about $10 \mathrm{Myr}$, this cusp is probably related to the star formation and/or molecular cloud fragmentation, and not the product of dynamical evolution. A possible conclusion is that $\mathrm{Cr} 197$ and vdB 92 deviate critically from dynamical equilibrium, and similar to the equally young NGC 2244 and Bochum 1 (of comparable mass), and Pismis 5 and NGC 1931 (of significantly lower mass), they are heading towards dissolution. This interpretation is also consistent with the super-virial state of both clusters (as well as the dissolving OC NGC 2244), which have velocity dispersions that are much higher than the $\sigma_{\mathrm{v}} \approx 1 \mathrm{~km} \mathrm{~s}^{-1}$ expected for nearly virialised clusters of similar mass and size as $\mathrm{Cr} 197$ and vdB 92. In this context, $\mathrm{Cr} 197$ and vdB 92 may be taken as a link between embedded clusters and young stellar associations.

We provide evidence that early star cluster dissolution may be detected, for instance, by means of important and systematic irregularities in the stellar radial density profile of clusters as massive as several $10^{2} M_{\odot}$. Studies like the present one are important for a better understanding of the crucial early evolution of embedded star clusters - and the dependence on mass and environment - that rarely result in a classical bound OC or, more frequently, lead to their dissolution into the field.

Acknowledgements. We thank the referee, Simon Goodwin, for interesting comments and suggestions. We acknowledge support from the Brazilian Institution CNPq. This publication makes use of data products from the Two Micron All Sky Survey, which is a joint project of the University of Massachusetts and the Infrared Processing and Analysis Centre/California Institute of Technology, funded by the National Aeronautics and Space Administration and the National Science Foundation. This research has made use of the WEBDA database, operated at the Institute for Astronomy of the University of Vienna.

\section{References}

Allison, R. J., Goodwin, S. P., Parker, R. J., et al. 2009, ApJ, 700, L99

Alter, G., Balazs, B., Ruprecht J., \& Vanysek, J. 1970, in Catalogue of Star Clusters and Associations, Budapest Akademiai Kiado, 2nd edition, ed. G. Alter, B. Balazs, \& J. Ruprecht

Bastian, N., Gieles, M., Lamers, H. J. G. L. M., Scheepmaker, R. A., \& de Grijs, R. 2005, A\&A, 431, 905

Battinelli, P., \& Capuzzo-Dolcetta, R. 1991, MNRAS, 249, 76

Battinelli, P., Brandimarti, A., \& Capuzzo-Dolcetta, R. 1994, A\&AS, 104, 379 van den Bergh, S. 1966, AJ, 71, 990

Bessel, M. S., \& Brett, J. M. 1988, PASP, 100, 1134

Bica, E., Bonatto, C., Barbuy, B., \& Ortolani, S. 2006, A\&A, 450, 105

Bica, E., Bonatto, C., \& Dutra C. 2008a, A\&A, 489, 1129

Bica, E., Bonatto, C., \& Camargo, D. 2008b, MNRAS, 385, 349

Bonatto, C., \& Bica, E. 2005, A\&A, 437, 483

Bonatto, C., \& Bica, E. 2006, A\&A, 455, 931

Bonatto, C., \& Bica, E. 2007, MNRAS, 377, 1301

Bonatto, C., \& Bica, E. 2008, A\&A, 477, 829

Bonatto, C., \& Bica, E. 2009a, MNRAS, 392, 483

Bonatto, C., \& Bica, E. 2009b, MNRAS, 394, 2127

Bonatto, C., \& Bica, E. 2009c, MNRAS, 397, 1915

Bonatto, C., Bica, E., \& Girardi, L. 2004, A\&A, 415, 571

Bonatto, C., Bica, E., Ortolani, S., \& Barbuy, B. 2006a, A\&A, 453, 121

Bonatto, C., Santos, Jr. J. F. C., \& Bica E. 2006b, A\&A, 445, 567

Cardelli, J. A., Clayton, G. C., \& Mathis, J. S. 1989, ApJ, 345, 245

Chen, L., de Grijs, R., \& Zhao, J. L. 2007, AJ, 134, 1368

Clariá, J. J. 1974a, ApJ, 79, 1022

Clariá, J. J. 1974b, A\&A, 37, 229

Collinder, P. 1931, AnLun, 2, 1

Dutra, C. M., Santiago, B. X., \& Bica, E. 2002, A\&A, 383, 219

Friel, E. D. 1995, ARA\&A, 33, 381

Froebrich, D., Scholz, A., \& Raftery, C. L. 2007, MNRAS, 374, 399

Furlan, E., Watson, D. M., McClure, M. K., et al. 2009, ApJ, 703, 1964 
C. Bonatto and E. Bica: Collinder 197 and vdB 92: dissolving star clusters?

Ghez, A. M., Salim, S., Weinberg, N. N., et al. 2008, ApJ, 689, 1044 Gieles, M., Sana, H., \& Portegies Zwart, S. F. 2010, MNRAS, 402, 1750 Girardi, L., Bertelli, G., Bressan, A., et al. 2002, A\&A, 391, 195

Goodwin, S. P. 2009, Ap\&SS, 324, 259

Goodwin, S. P., \& Bastian, N. 2006, MNRAS, 373, 752

Gouliermis, D., Kontizas, M., Korakitis, R., et al. 2000, AJ, 119, 1737

de Grijs, R., \& Goodwin, S. P. 2008, MNRAS, 383, 1000

de Grijs, R., \& Goodwin, S. P. 2009, in IAU Symp., 256, ed. J. T. van Loon, \&

J. M. Oliveira (Cambridge Univ. Press)

de Grijs, R., Kouwenhoven, M. B. N., \& Goodwin, S. P. 2008, AN, 329, 972

Gum, C. S. 1955, MmRAS, 67, 155

King, I. 1962, AJ, 67, 471

Kroupa, P. 2001, MNRAS, 322, 231

Lada, C. J., \& Lada, E. A. 2003, ARA\&A, 41, 57

Lauberts, A. 1982, ESO/Uppsala survey of the ESO(B) atlas, European Southern Observatory

Magakian, T. Y. 2003, A\&A, 399, 141

Massey, P., Johnson, K. E., \& De Gioia-Eastwood, K. 1995, ApJ, 454, 151

Momany, Y., Zaggia, S., Gilmore, G., et al. 2006, A\&A, 451, 515
Naylor T., \& Jeffries R. D. 2006, MNRAS, 373, 1251

Pettersson, B., \& Reipurth, B. 1994, A\&ASS, 104, 233

Piskunov, A. E., Schilbach, E., Kharchenko, N. V., Röser, S., \& Scholz, R.-D. 2007, A\&A, 468, 151

Poetzel, R., Mundt, R., \& Ray, T. P. 1989, A\&A, 224, L13

Rodgers, A. W., Campbell, C. T., \& Whiteoak, J. B. 1960, MNRAS, 121, 103

Siess, L., Dufour, E., \& Forestini, M. 2000, A\&A, 358, 593

Skrutskie, M., Schneider, S. E., Stiening, R., et al. 1997, in The Impact of Large Scale Near-IR Sky Surveys, ed. F. Garzon et al. (Netherlands: Kluwer), 210, 187

Soares, J. B., \& Bica, E. 2003, A\&A, 404, 217

Spitzer, L. 1987, in Dynamical Evolution of Globular Clusters (Princeton, NJ: Princeton University Press), 191

Trager, S. C., King, I. R., \& Djorgovski, S. 1995, AJ, 109, 218

Trippe, S., Gillessen, S., Gerhard, O. E., et al. 2008, A\&A, 492, 419

Tutukov, A. V. 1978, A\&A, 70, 57

Vogt, N., \& Moffat, A. F. J. 1973, A\&AS, 9, 97

Whitmore, B. C., Chandar, R., \& Fall, S. M. 2007, AJ, 133, 1067

Zacharias, N., Finch, C., Girard, T., et al. 2010, AJ, 139, 2184 